\title{
Development and characterization of a CRISPR/Cas9n-based multiplex genome editing system for Bacillus subtilis
}

\author{
Dingyu Liu ${ }^{\dagger}$, Can Huang ${ }^{\dagger}$, Jiaxin Guo, Peiji Zhang, Tao Chen, Zhiwen Wang ${ }^{*}$ (D) and Xueming Zhao
}

\begin{abstract}
Background: Metabolic engineering has expanded from a focus on designs requiring a small number of genetic modifications to increasingly complex designs driven by advances in multiplex genome editing technologies. However, simultaneously modulating multiple genes on the chromosome remains challenging in Bacillus subtilis. Thus, developing an efficient and convenient method for B. subtilis multiplex genome editing is imperative.

Results: Here, we developed a CRISPR/Cas9n-based multiplex genome editing system for iterative genome editing in B. subtilis. This system enabled us to introduce various types of genomic modifications with more satisfying efficiency than using CRISPR/Cas9, especially in multiplex gene editing. Our system achieved at least 80\% efficiency for 1-8 kb gene deletions, at least $90 \%$ efficiency for 1-2 kb gene insertions, near 100\% efficiency for site-directed mutagenesis, 23.6\% efficiency for large DNA fragment deletion and near 50\% efficiency for three simultaneous point mutations. The efficiency for multiplex gene editing was further improved by regulating the nick repair mechanism mediated by ligD gene, which finally led to roughly $65 \%$ efficiency for introducing three point mutations on the chromosome. To demonstrate its potential, we applied our system to simultaneously fine-tune three genes in the riboflavin operon and significantly improved the production of riboflavin in a single cycle.
\end{abstract}

Conclusions: We present not only the iterative CRISPR/Cas9n system for B. subtilis but also the highest efficiency for simultaneous modulation of multiple genes on the chromosome in B. subtilis reported to date. We anticipate this CRISPR/Cas9n mediated system to greatly enhance the optimization of diverse biological systems via metabolic engineering and synthetic biology.

Keywords: CRISPR/Cas9n, Multiplex genome editing, Combinatorial metabolic engineering, Riboflavin, Nick repair mechanism

\section{Background}

Metabolic engineering for the production of chemicals, fuels, and pharmaceuticals typically requires extensive modulation of metabolic networks to enhance the productivity of the host strains $[1,2]$. The expression levels of pathway genes play a key role in determining

\footnotetext{
*Correspondence: zww@tju.edu.cn

${ }^{\dagger}$ Dingyu Liu and Can Huang contributed equally to this work

Frontier Science Center for Synthetic Biology and Key Laboratory of Systems Bioengineering (Ministry of Education), SynBio Research Platform, Collaborative Innovation Center of Chemical Science and Engineering (Tianjin), Department of Biochemical Engineering, School of Chemical Engineering and Technology, Tianjin University, Tianjin 300072, China
}

the production of metabolites in an organism [3]. The toxic intermediates may accumulate with imbalanced expression of pathway genes, which can significantly negatively affect cell growth [4]. The construction of balanced metabolic pathways is consequently one of the most important research subjects of metabolic engineering and synthetic biology. The metabolic engineering 'toolbox' has expanded from a focus on individual genetic modifications to increasingly complex designs that require the modulation of multiple genes throughout the metabolic network of the cell [5-7]. To support this increased engineering complexity, powerfully 
iterative genetic engineering tools are increasingly being favored by metabolic engineers.

Bacillus subtilis, which was granted GRAS (generally regarded as safe) status by the US Food and Drug Administration, has long been widely used for the production of enzymes, drug precursors, platform compounds, biofuels and biopolymers [8]. It readily secretes products into the extracellular medium and can metabolize nearly any carbon source, making it an attractive biomanufacturing platform [9]. While B. subtilis is an ideal organism for metabolic engineering applications, the development of genetic tools is lagging behind popular production hosts such as Escherichia coli and Saccharomyces cerevisiae, especially in multiplex genome editing. In E. coli and S. cerevisiae, efficient genome editing tools and a range of established multiplex pathway-optimization techniques (MAGE [10], YOGE [11], TRMR [12], RAGE [13], CREATE [14], CHAnGE [15], and so on) have been developed, which enabled the simultaneous modification of several loci, and greatly enhanced the ability to engineer complex pathways. Although a substantial collection of counter-selectable markers [16-18], or $\lambda$-Red phage mediated singlestranded DNA recombination [19] tools are available for engineering $B$. subtilis, these techniques requires time-consuming sequential transformation steps and are unable to achieve highly efficient complex gene editing. The current genetic engineering tools, therefore, still represent a bottleneck for multiple genes modulation in B. subtilis.

Recently, microbial genome editing techniques have progressed significantly due to the extensive research conducted on the CRISPR system (clustered regularly interspaced short palindromic repeats), derived from the RNA-guided immune systems found in many bacteria and archaea $[1,20]$. The introduction of the CRISPR/Cas9 system not only eliminated the need for selection markers in genome editing but also dramatically increased the editing efficiency [21-24]. Current genome editing applications based on the type II-CRISPR/Cas9 system in bacteria are classified into two categories: Cas9-mediated genome editing and Cas9 nickase (Cas9n)-mediated genome editing [25]. SpCas9 and SpCas9n induce the integration of the repair template into the target locus at similar frequencies. However, compared to Cas9-mediated genome editing methods, Cas9n causes less damage and toxicity to the host [26]. At the same time, the singlestrand nick created by Cas9n is highly suitable for repair and thus improves the genome manipulation efficiency $[27,28]$. CRISPR/Cas9n assisted genome editing tools have recently been developed for a number of bacteria, including E. coli [29], Lactobacillus reuteri [30], Clostridium sp. [31] and Bacillus licheniformis [32].
In B. subtilis, the CRISPR/Cas9 methodology has been established to introduce gene insertions, deletions and replacements [33-35]. Furthermore, double-target editing was achieved and reached high efficiency through the optimization of various editing template parameters and PAM sites [34]. However, a complicated experimental procedure had to be developed to facilitate this technique. Moreover, multiplex site-editing (more than two sites) using CRISPR/Cas9 was not feasible due to the low recombination efficiency and toxicity of the multiple Cas9-induced DSBs (double-strand breaks). Thus, it is necessary to develop a simple technique to simultaneously modulate multiple genes on the chromosome with high efficiency.

In this research, we developed a CRISPR/Cas9n-mediated genome editing technique for $B$. subtilis. Compared to recently reported CRISPR/Cas9-based genome modification systems, the Cas9n-mediated editing technique showed higher efficiency for large genomic deletions and multiplex editing. Subsequently, CRISPR/Cas9nmediated multiplex gene editing was further improved by inhibiting nicks re-ligation. As a demonstration, the CRISPR/Cas9n-mediated multiplex genome editing was applied to generate a combinatorial RBS library of the riboflavin operon, in which three genetic loci were simultaneously modulated to improve the strain's riboflavin production capacity.

\section{Results and discussions}

\section{Investigation of the CRISPR/Cas-induced nickase system} in B. subtilis

Recently, Cas9n-mediated genome editing has been shown to be an efficient and precise tool in a number of bacteria [30-32, 36, 37]. The nickase is an effective tool to circumvent DSB-induced lethality and the repair mechanism allows the resulting nicks to trigger HDR (homology directed repair) with less toxicity for the host cells [27]. In contrast to the DSB induced by native Cas 9 , the singlestrand nick created by Cas9 nickase is more suitable for repair and thus improves the genome manipulation efficiency $[27,28]$. We, therefore, exchanged the native Cas9 of existing constructs with Cas9n for use in B. subtilis, a substitution that may lead to higher survival frequencies of transformed cells and better editing efficiency.

To confirm that CRISPR/Cas9n was functional in $B$. subtilis, we tested different mutant versions of Cas9 without a repair template. In parallel, WT Cas9 was used as control. Cas9 and Cas9n (D10A and H840A) were individually introduced into $B$. subtilis together with coexpression of the target gRNA ( $a m y E$ ). The total colony forming units (CFU) were calculated by counting the corresponding transformants on agar plates (Fig. 1). The CFU number was reduced significantly due to the 


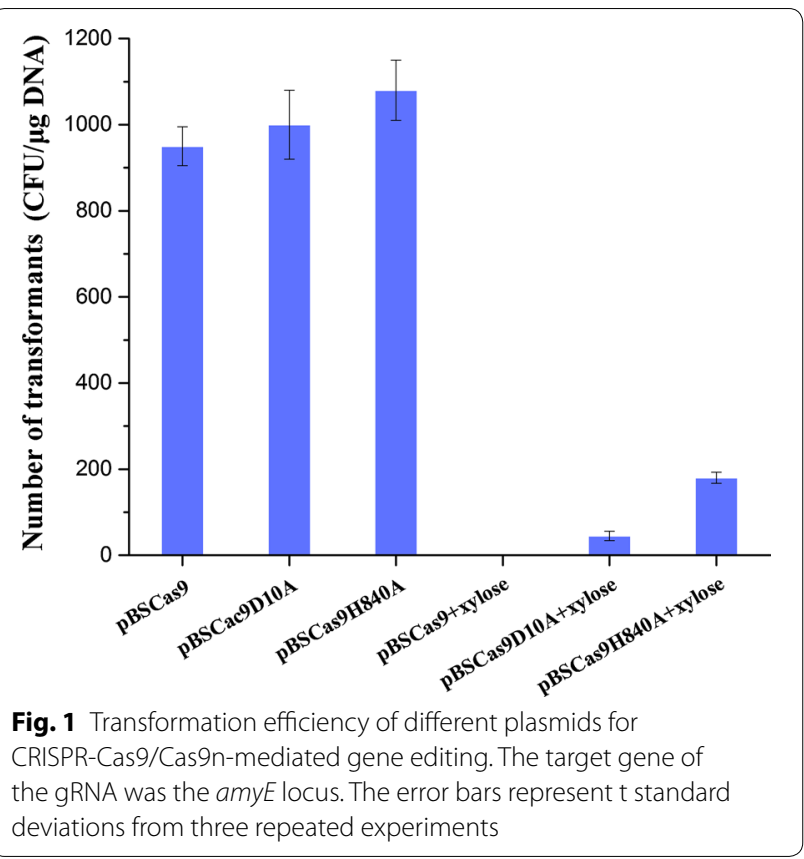

induced expression of Cas9/Cas9n, indicating that Cas9/ Cas9n had most likely cleaved the genome as a consequence of the presence of the gRNA directed against $a m y E$. By contrast, the transformation reached an appreciable frequency $\left(\sim 10^{3} \mathrm{CFU} / \mu \mathrm{g}\right.$ DNA $)$ when Cas9/Cas9n were not induced. These efficiencies of plasmid transformation were less than the average levels observed for $B$. subtilis, which may be due to leaky expression of Cas9/ Cas9n from the induced promoter PxylA.

In this work, the transformation efficiencies with induced Cas9n were appreciably higher than with induced Cas9, and those of Cas9nH840A were higher than the efficiencies of Cas9nD10A. The transformation efficiency with induced Cas9nD10A was fourfold lower than that of Cas9nH840A (Fig. 1). These data indicated that Cas9n was functional in B. subtilis and Cas9nD10A had most likely cleaved the genome with higher efficiency than Cas9nH840A. Consequently, Cas9D10A nickase was employed in our CRISPR-Cas9n genome editing system, and Cas9n refers to the Cas9D10A mutant in further text.

\section{Establishment of CRISPR/Cas9n-mediated genome editing in B. subtilis}

Recently, several CRISPR/Cas systems for B. subtilis have been developed using different design strategies, including single-plasmid systems [33, 38], two-plasmid systems [35] and chromosomal maintenance system [34]. Our CRISPR/Cas9n-mediated genome editing system is an improved two-plasmid system (Fig. 2a), which is composed of a vector encoding the CRISPR components and another vector carrying the donor DNA. The induced Cas9n expression system and gRNA constitutive expression cassettes were assembled on the CRISPR components plasmid (pBSCas9n), which also has a thermo-sensitive replication origin and a cat gene. The plasmids derived from pDonor, harboring the donor DNA, were designed to generate gene deletions, insertions, or replacements while altering the PAM sequences to allow mutant cells to escape CRISPR induced cell death. To construct the plasmid curing system, we used the mannose inducible promoter Pmanp to express a gRNA targeting the replication origin gene rep60 on the donor DNA plasmid. For iterative genome editing, the rep60-targeting gRNA was expressed, which induced Cas9n to cleave the gRNA plasmid, resulting in plasmid elimination.

In the reported two plasmid systems of B. subtilis, gRNA and donor DNA were assembled in the same plasmid [35]. Our system separates the gRNA and donor DNA onto different vectors, which makes it easy and fast to complete the required plasmid construction, especially when assembling multiple gRNAs and donor DNAs. Compared to the single-plasmid and chromosomal maintenance systems, the two-plasmid system has the advantages of being suitable for complex modifications such as large fragment insertion and multiplex locus editing, as well as fast operation with high efficiency [39]. In this study, the optimized iterative editing system enjoyed the added advantage of modular construction protocols, reduced time consumption and increased convenience, which makes it more applicable to multiplex genome editing.

Each cycle of editing starts with the successive introduction of the plasmids pBSCas9n and pDonor into the cells. After inducing the expression of Cas9n, the hosts' genome is cleaved by CRISPR-mediated digestion unless mutations were acquired at the PAM sequences. Spreading the induced cells on medium containing chloramphenicol and erythromycin allowed the selection of cells containing the desired modification. Subsequently, correct mutants were incubated for plasmid curing and then analyzed for antibiotic sensitivity to confirm the loss of the plasmid, after which single colonies were grown to prepare competent cells for the next round of editing. The time required for each editing cycle is 3 days (Fig. 2b).

\section{Characterization of CRISPR/Cas9n mediated genome editing in $B$. subtilis}

The type II CRISPR-Cas9 system has been proven to be suitable for introducing various genome editing in B. subtilis, and was applied in the fields of metabolic 


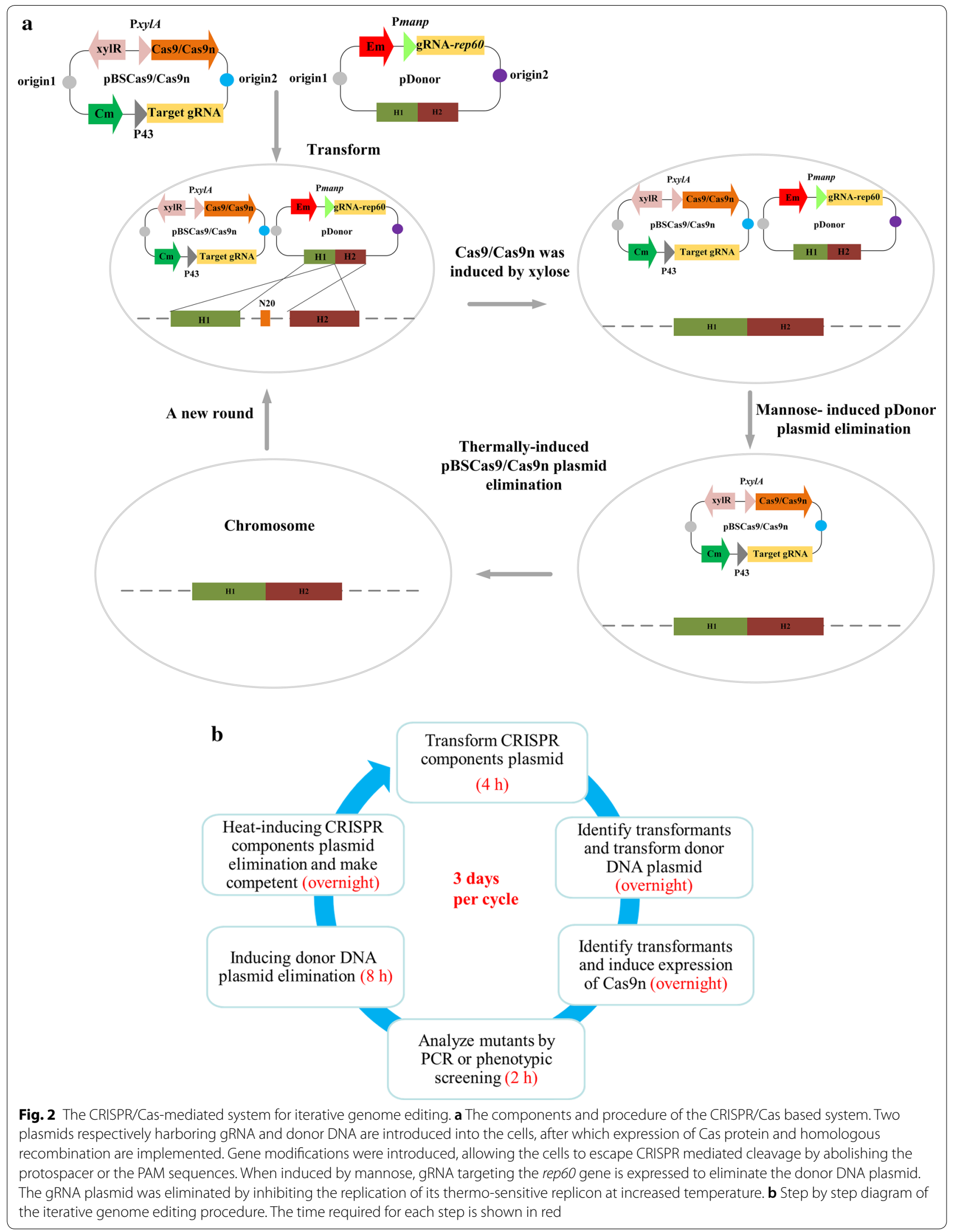


engineering and synthetic biology [39]. Nevertheless, CRISPR/Cas9-mediated genome editing is still at the exploratory stage, especially because the necessary DSB caused by Cas9 may require longer incubation times for repair, or may even preclude the survival of cells after transformation with the CRISPR/Cas9 system. In this work, the alternative CRISPR/Cas9n-mediated genome editing system was established and characterized in $B$. subtilis, with the aim to overcome existing shortcomings of the classical CRISPR/Cas9 system. We, therefore, quantitatively compared and analyzed the effect of CRISPR/Cas9 and CRISPR/Cas9n on editing efficiency.

We first systematically evaluated the ability of CRISPRCas9/Cas9n mediated gene editing to introduce various types of modifications including gene deletions, insertions and deletions of large-scale chromosomal regions (Fig. 3). As shown in Fig. 3a, our system yielded at least $90 \%$ editing efficiency for 1-4 kb gene deletions with both CRISPR/Cas9 and CRISPR/Cas9n. However, the efficiency for $6 \mathrm{~kb}$ and $8 \mathrm{~kb}$ gene deletions with Cas9 as editing tool decreased dramatically in our system. By contrast, the efficiency with Cas9n remained above $80 \%$. The CRISPR/Cas9n system produced $98 \%$ correct $1 \mathrm{~kb}$ gene insertions and $92 \% 2 \mathrm{~kb}$ gene insertions, which was better than the results obtained with CRISPR/Cas9 (95\% and $87 \%$, respectively) (Fig. 3b). Moreover, the ability of the CRISPR/Cas9n system to introduce large genomic deletions was slightly better than that of CRISPR/Cas9 (Fig. 3c). Large DNA fragment deletions remain problematic with the traditional genome editing method. Recent large genomic deletion studies have reported low mutation efficiency [40] or were only feasible in specific strains containing antibiotic resistance markers [41]. Recently, CRISPR/Cas9n-based dual-targeted nicks have been used to delete $25.1 \mathrm{~kb}$ fragment from the genome of B. subtilis with the efficiency of 53\% [37]. In this study, a prophage (-like) regions, a large DNA fragment of $20.5 \mathrm{~kb}$ was completely deleted using CRISPR/Cas9n. Although the deletion efficiency for large fragment only reached $23.6 \%$ by our CRISPR/Cas9n system, only one nick was targeted in this study. This operation was simple and successful, and the efficiency was higher than our previous marker-free strategy [16].

In addition to the advantages in editing efficiency, the CFU number in almost all the experiments with CRISPR/ Cas9n as editing tool were higher than with CRISPR/ Cas9 (Fig. 3a-c), indicating a reduced toxicity of the former to the host cells in the process of genome editing. Hence, the CRISPR/Cas9n system was more fault-tolerant in practical molecular cloning operations.

To our best knowledge, the CRISPR system based on Cas9n-induced SSBs (single-strand breaks) has not been previously characterized systematically in B. subtilis.
Hence, this is the comprehensive report of the successful use of such a system in B. subtilis. In our editing system, Cas9-mediated genome modification achieved a similar efficiency to previous reports of CRISPR/Cas9 system for B. subtilis [33-35, 38]. However, Cas9n causes less damage to the host than the CRISPR/Cas9 genome modification system and allows for more precise genome editing [36, 42]. Based on our results, the CRISPR/Cas9n system constructed in this study can be applied to $1-6 \mathrm{~kb}$ gene deletions and 1-2 kb gene insertions using fixed-length homology arms (500 bp) with at least $90 \%$ efficiency. Furthermore, the Cas9n-based system has the advantage of long fragment deletion with higher efficiency. This is because the single-strand nick created by Cas9n is prone to homologous recombination repair and thus improves the genome editing efficiency $[27,43]$. In addition to the higher editing efficiency, more CFU could be obtained by genome editing with CRISPR/Cas9n. The positive clones are thus easier to obtain in practice, especially when editing genes associated with cell growth.

\section{Multiplex genome editing mediated by CRISPR/Cas9n system}

The introduction of genomic point mutations is widely used strategy in metabolic engineering, including sitedirected mutagenesis, RBS optimization, and so on. The recently developed CRISPR/Cas9 based point mutation systems for B. subtilis reached up to $100 \%$ editing efficiency for single-point mutations [35]. However, the efficiency for two simultaneous mutations decreased dramatically, and the modification of three sites was not attempted in B. subtilis, suggesting a limitation for combinatorial modulation. DSB based on Cas9 is a powerful counter-selection marker that ensures the high apparent editing rate among the obtained colonies. However, it is so strong that the cell survival rate decreases dramatically as the number of target locis increase. Multiple genomic cleavage sites based on Cas9 are more difficult to repair by HDR because more breaks and steric hindrance might arise with the increase in the number of target loci based on DSB (Fig. 4a). In contrast to the DSB based Cas9, nickase can facilitate homology-directed repair with minimal off-site mutagenic activity [26]. The genome is considered an integrated structure, although it can be converted into cyclic annular nicked DNA by the action of nickase (Fig. 4a). Nicked genomic DNA is typically repaired either seamlessly or through high-fidelity HDR, and it is easier to repair than cleaved genomic DNA containing DSB. Thus, we presumed that the CRISPR/Cas9n system enables more efficient introduction of multiplex point mutations for gene editing.

Based on this hypothesis, we explored the ability of our CRISPR-based system to introduce single and several 

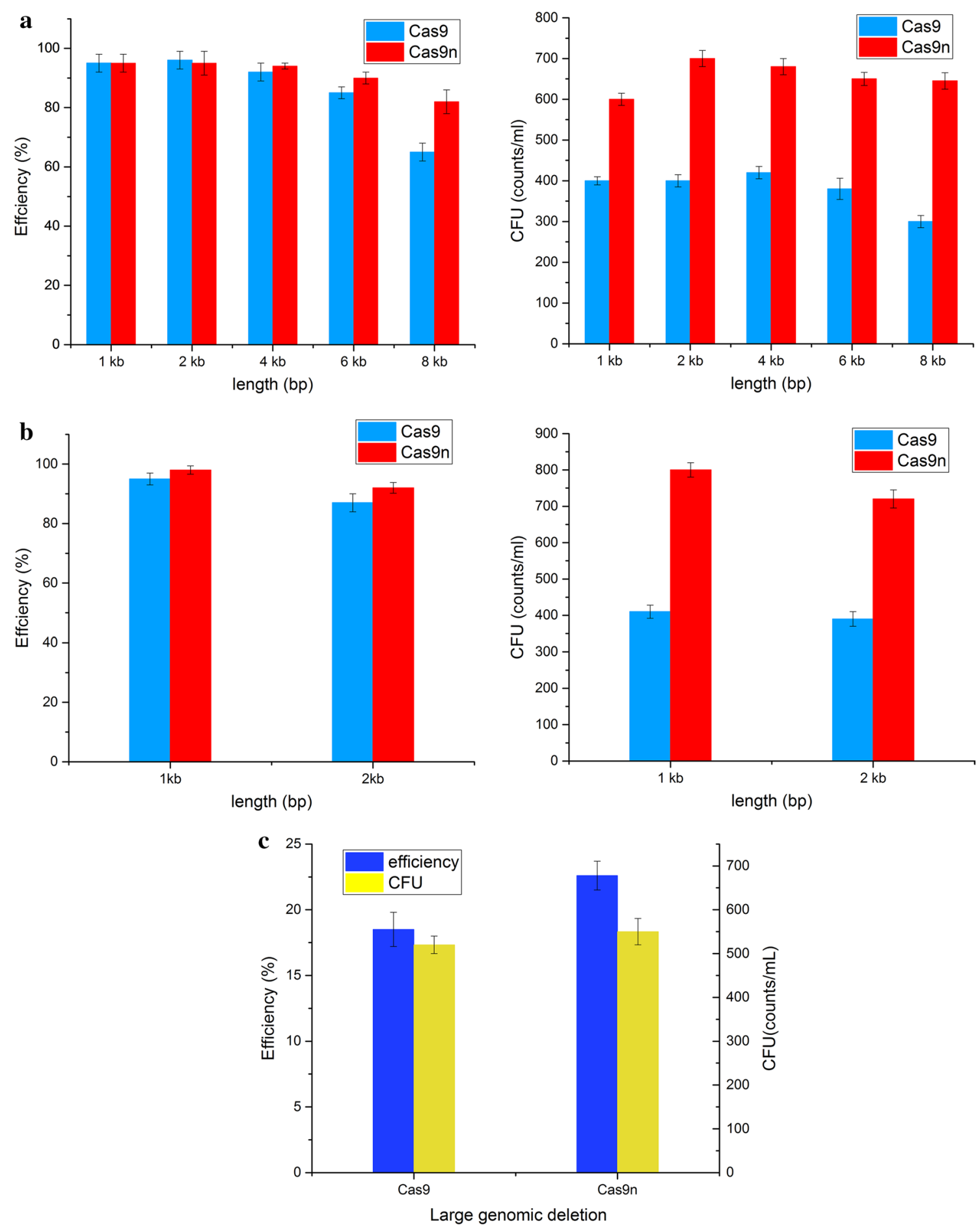

Fig. 3 Characterization of CRISPR-Cas9/Cas9n mediated genome editing. a Editing efficiency and CFU for sequence deletions using CRISPR/Cas9 or CRISPR/Cas9n. For deletion, the region of the amyE gene and flanking sequences were deleted. $\mathbf{b}$ Editing efficiency and CFU for gene insertions. A deleted sequence in the amyE region was restored. $\mathbf{c}$ Editing efficiency and CFU for large genomic deletions. A prophage (-like) regions was deleted. In these genetic modifications, 500 bp homologous-arms were used for recombination. All error bars represent the value of standard deviation which were calculated from three repeated experiments 
CRISPR/Cas9 Mediated Multiplex Breaks

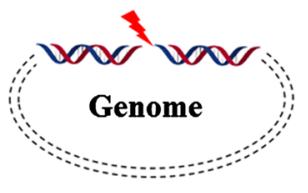

Single site

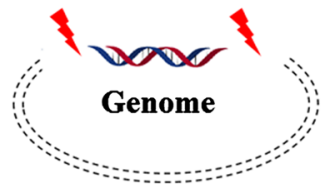

Double sites

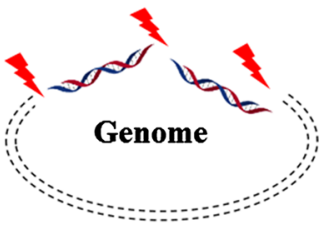

Multiplex sites

CRISPR/Cas9n Mediated Multiplex Breaks

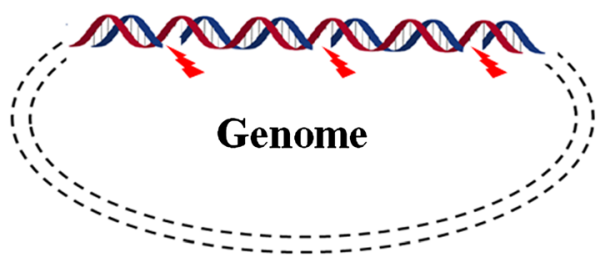

b

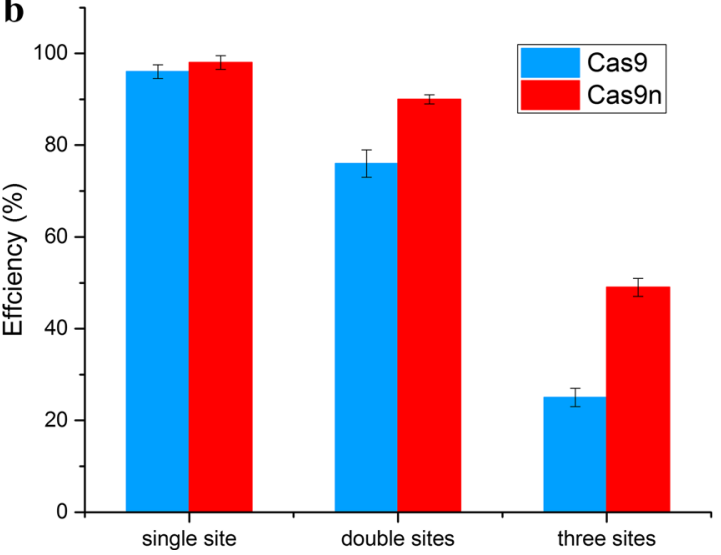

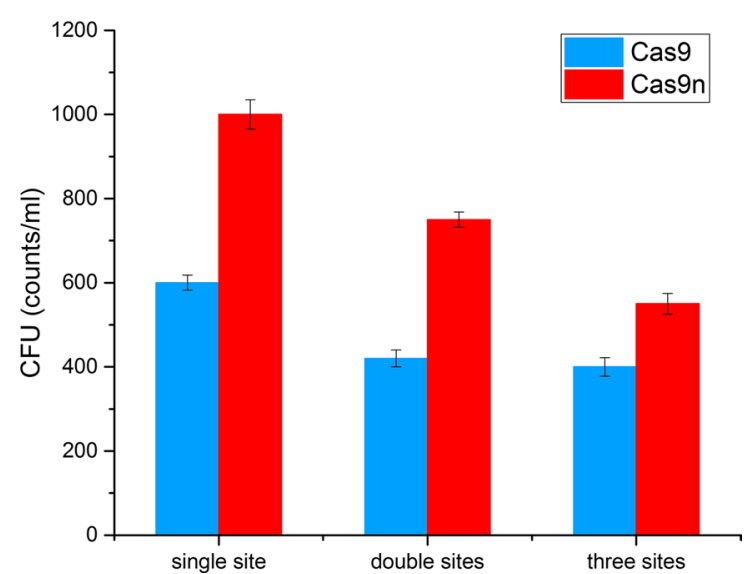

Fig. 4 Strategy for CRISPR-Cas9/Cas9n mediated multiplex point mutations. a Assumed mechanism of CRISPR-Cas9/Cas9n mediated multiplex breaks. b Editing efficiency and CFU for multiplex point mutations using the CRISPR-Cas9/Cas9n system. In these genetic modifications, 500 bp homologous-arms were used to achieve recombination. All error bars represent the value of standard deviation which were caculated from three repeated experiments

simultaneous point mutations. We targeted three sites, $a m y E$, upp, and sigE, and designed donor DNAs to introduce codon replacements in these target genes. The two plasmids harboring the corresponding gRNAs and donor DNAs were designed and constructed in a modular fashion. Two CRISPR systems were evaluated by introducing codon replacements in the PAM sequences. The results showed that single-point mutations could be introduced with near $100 \%$ efficiency using either CRISPR/Cas9 or CRISPR/Cas9n. However, increasing the number of target sites in the CRISPR/Cas9 system, significantly decreased the editing efficiency, with only $19.5 \%$ correct clones for three-point mutations. In contrast to CRISPR/ Cas9, the CRISPR/Cas9n system maintained a more desirable editing efficiency for multiplex genome editing, with $90 \%$ efficiency for two mutations and $49 \%$ efficiency for three mutations (Fig. 4b).

We employed the CRISPR/Cas9n methodology to overcome some deficiencies of the DSB approach and introduce multiplex mutations. To our best knowledge, the $49 \%$ observed in this study is the highest efficiency for simultaneous modulation of multiple genes on the chromosome in B. subtilis reported to date. Recently, a CRISPR/Cas9-facilitated multiplex pathway optimization technique was developed in $E$. coli, by co-expressing $\lambda$-Red recombineering system and Cas 9 system, which yielded $70 \%$ efficiency for modulating three target loci simultaneously [44]. However, a similar CRISPR/Cas9medited system could not achieve satisfying efficiency for multiplex pathway modification in B. subtilis. We 
inferred that the low efficiency of multiplex mutations is due to the comparatively inefficient HDR without expressing an exogenous recombination system and the low cell survival rate due to the multiple Cas9-induced DSBs. Furthermore, the low plasmid transformation efficiency of $B$. subtilis compared to $E$. coli is also a reason for the low multiplexing efficiency. The determination of the positions of the PAM sequences and design of gRNAs greatly influenced the efficiency of CRISPR-based modulation [33, 45, 46]. The expression of an exogenous recombination system and optimization of gRNAs may further boost the frequency of homologous recombination and thus contribute to the efficiency of CRISPR/ Cas9n mediated gene editing. In addition, regulation of the nick repair mechanism may improve CRISPR/Cas9n mediated genome editing.

\section{Improving CRISPR/Cas9n mediated multiplexing by inhibiting nicks re-ligation in $B$. subtilis}

Although CRISPR/Cas9n-medited multiplex point mutations could be introduced with excellent efficiency, there was a certain number of false-positive colonies in the process of gene editing. Nicks are efficiently re-ligated and this would be predicted to compete with homologous recombination to reduce the efficiency of gene editing [26, 27]. We suspected that false-positive colonies arose from cells whose genome had not been cleaved and from cells that have been repaired by the non-HDR mechanism. Therefore, the non-HDR repair mechanism of Cas9n-induced SSB should be suppressed to improve the editing efficiency based on HDR repair.

The re-ligation of nicks depends on DNA ligases, including $\mathrm{NAD}^{+}$-dependent ligase (encoded by gene ligA) and ATP- dependent ligase (encoded by gene ligD) in $B$. subtilis. The gene ligA is challenging to engineer because it is an essential gene of $B$. subtilis, which is associated with DNA replication and repair. By contrast, the gene $l i g D$, whose corresponding protein uses ATP to form a phosphodiester at the site of a SSB and catalyze DNA ligation [47], is an appropriate target in regulation of nicks re-ligation in $B$. subtilis. The underlying mechanism is similar to the NHEJ (non-homologous end joining) pathway, which is one of the major mechanisms for repairing strand breaks that occur in genomic DNA [48] (Fig. 5a). Thus, the ligD gene was targeted to regulate the SSB repair pathway. We presumed that knocking out $l i g D$ would further improve the efficiency of multiplex genome editing (Fig. 5b).

Firstly, the function of $\operatorname{lig} D$ was characterized and investigated in B. subtilis harboring CRISPR-Cas9/Cas9n system. It was overexpressed from plasmid pHP13 to repair Cas9/Cas9n-induced genome cleavage. As shown in Fig. 6a, cell growth of strains P5 and P6 was severely affected by the Cas9/Cas9n-induced genomic cleavage. By contrast, cell growth of strains P1 and P2 was dramatically improved by enhancing the repair of nicks via overexpression of ligD. Moreover, knockout of ligD in wild type B. subtilis 168 had no obvious influence with cell growth (Additional file 2: Figure S4). We next assessed the efficiency of CRISPR/Cas9n-induced multiplex point mutations in B. subtilis $168 \Delta$ ligD. The same sites as before were targeted to introduce codon replacements. As shown in Fig. 6b, CRISPR/Cas9n-mediated multiplex gene editing was further enhanced, reaching $91 \%$ efficiency for two-point mutations and 65\% efficiency for three-point mutations. Thus, the improved CRISPR/ Cas9n system achieved higher efficiency for the simultaneous modification of several loci, which enables the implementation of multiplex metabolic engineering on the chromosome of B. subtilis.

For the first time, we employed the ligD mediated repair mechanism to improve CRISPR/Cas9n mediated multiplex genome editing. The efficiency for simultaneous modulation of three sites was further increased to $65 \%$. The ligD gene, which is involved in NHEJ repair and related pathways, is a stable expressed and nonessential in the B. subtilis. It should be noted that NHEJ does not work efficiently or is not present in most bacteria, but Bacillus species possess a conserved prokaryotic NHEJ pathway that is essential for repair of strand breaks arising in the stationary phase and spore dormancy period $[49,50]$. In essence, the re-ligation of strand breaks is mediated by a two-component $\mathrm{Ku}$-ligase break repair complex. The knockout of ligD facilitated the repair of HDR mediated strand breaks by inhibiting the nicks religation mechanism (Fig. 5). This type of DNA ligasebased repair mechanism should be paid attention to the research of genome editing for Bacillus species.

\section{Application of CRISPR/Cas9n-mediated multiplex genome editing for combinatorial pathway modulation}

To demonstrate the applicability of CRISPR/Cas9n-mediated multiplexing, genetic components of the B. subtilis riboflavin operon were modulated in a combinatorial fashion to optimize the metabolic efficiency of riboflavin biosynthesis. The plasmid library pDonor-ribRBSLib provided the donor DNAs for homologous recombination, encompassing three modulation libraries for the simultaneous regulation of the $r i b B$, ribA, and ribH genes. The corresponding gRNA plasmid pBSCas9n-gRNArib, which functioned to target the Cas9n protein to the regulator sequences of $r i b B, r i b A$ and $r i b H$, was constructed to encode three gRNAs with their N20 sequences complementary to the native RBS regions of these genes.

In B. subtilis, the riboflavin biosynthesis genes, ribG, ribB, $r i b A$ and $r i b H$, are clustered in a single operon, the 
a

ligD-mediated nick re-ligation

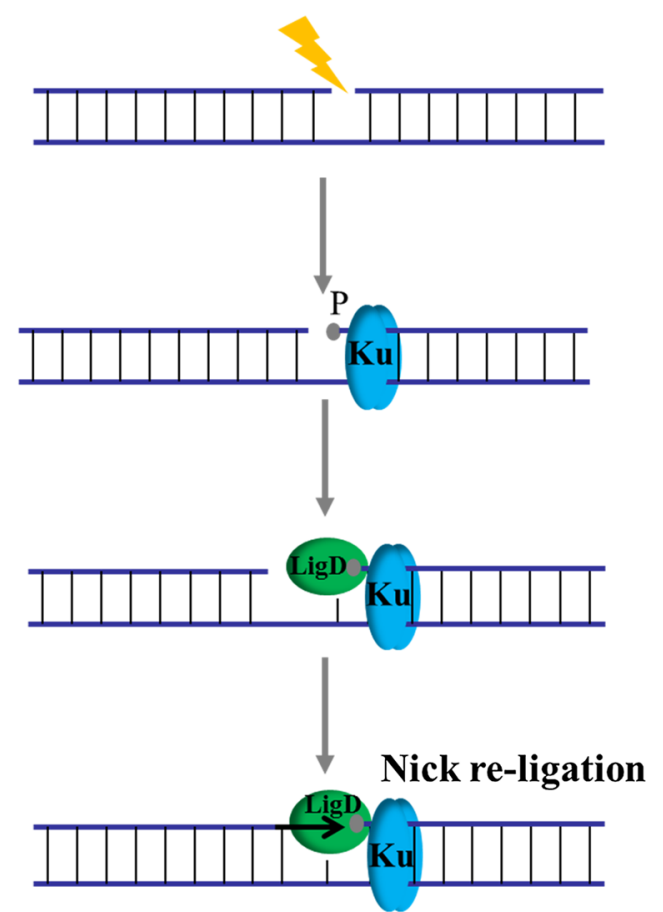

b

Predominantly HDR repair in $\Delta l i g D$

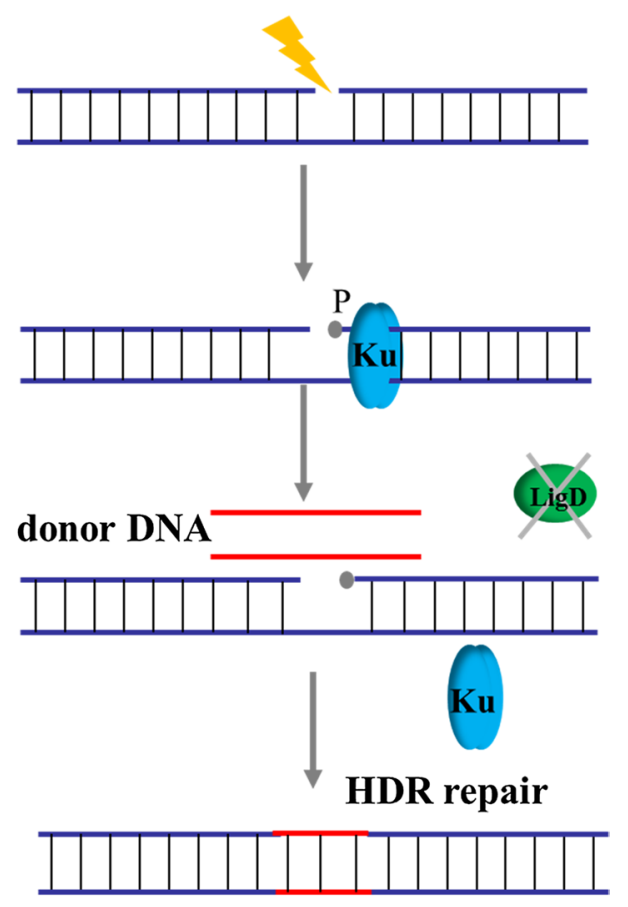

Fig. 5 Mechanism of ligD mediated nick ligation in B. subtilis. a The ligD-mediated nick re-ligation. A Ku homodimer binds to the end of the DNA break and recruits LigD protein. The polymerase domain of LigD specifically binds to a 5'-phosphate (P) and promotes end-synapsis-, together with protein $\mathrm{Ku}$. The nuclease and polymerase activities of LigD, and possibly other factors, process the break termini, if required, to restore complementary ends. Finally, ligation of the nick by LigD repairs the break. $\mathbf{b}$ Predominantly HDR repair in $\triangle$ ligD. When LigD is repressed, the DNA break is repaired by the HDR mechanism

structure of which was studied thoroughly (Fig. 7a). In our previous study, strain BS89 was constructed for riboflavin production by deregulating rib operon via overexpression of $r i b A$ [51]. In this study, to further deregulate the $r i b$ operon, three genes ( $r i b B$, ribA and $r i b H)$ involved in riboflavin biosynthesis were engineered through combinatorial modulation of their RBS to balance and optimize their expression for improved riboflavin production.

To achieve the desired efficiency for multiple modulations, BS89 was engineered by deleting ligD to generate BS89 4 ligD. The plasmids pBSCas9n-gRNArib and pDonor-ribRBSLib were introduced into BS89 $\operatorname{lig} D$ to initiate homologous recombination to replace the native RBS regions of ribB, ribA and ribH. The $B$. subtilis library carrying two plasmids was incubated for $8 \mathrm{~h}$ in medium with $1 \%$ xylose. The cultured library was diluted and spread on LB agar plates containing appropriate antibiotics. The colonies were preliminarily assessed by visually screening yellow color. Nearly 200 yellow colonies were selected, and these strains were characterized in 96-well plate fermentations. Eventually, 190 strains were cultivated to produce riboflavin in the 96-well plate (Fig. $7 \mathrm{~b}$ ).
Compared to BS89, 111 strains were improved in terms of riboflavin production. DNA sequencing was employed to analyze the 20 colonies with the highest production using the primer pairs P1-F/L, P2-F/L and P3-F/L. Based on the DNA sequence analysis, 11 colonies had modulations in all three genetic targets, while the other had two modulations genes. According to the DNA sequencing data, the multiplex library had good diversity for metabolic optimization. To analyze the production performance of the strains systematically and comprehensively, the yields of the 50 most productive strains were determined (Fig. 7c).

In this work, the efficiency for three-point mutations was about $50 \%$, which was lower than the expected efficiency (65\%). In practice, the multiplexing efficiency was limited by several genome editing factors, specifically the PAM site sensitivity and relative position of selected targets. It has been observed that certain PAM sites are less susceptible to CRISPR system-mediated strand breaks since the editing efficiency can vary substantially between PAM sites in a single gene [52]. The problem in gRNA design may be associated with the formation of 

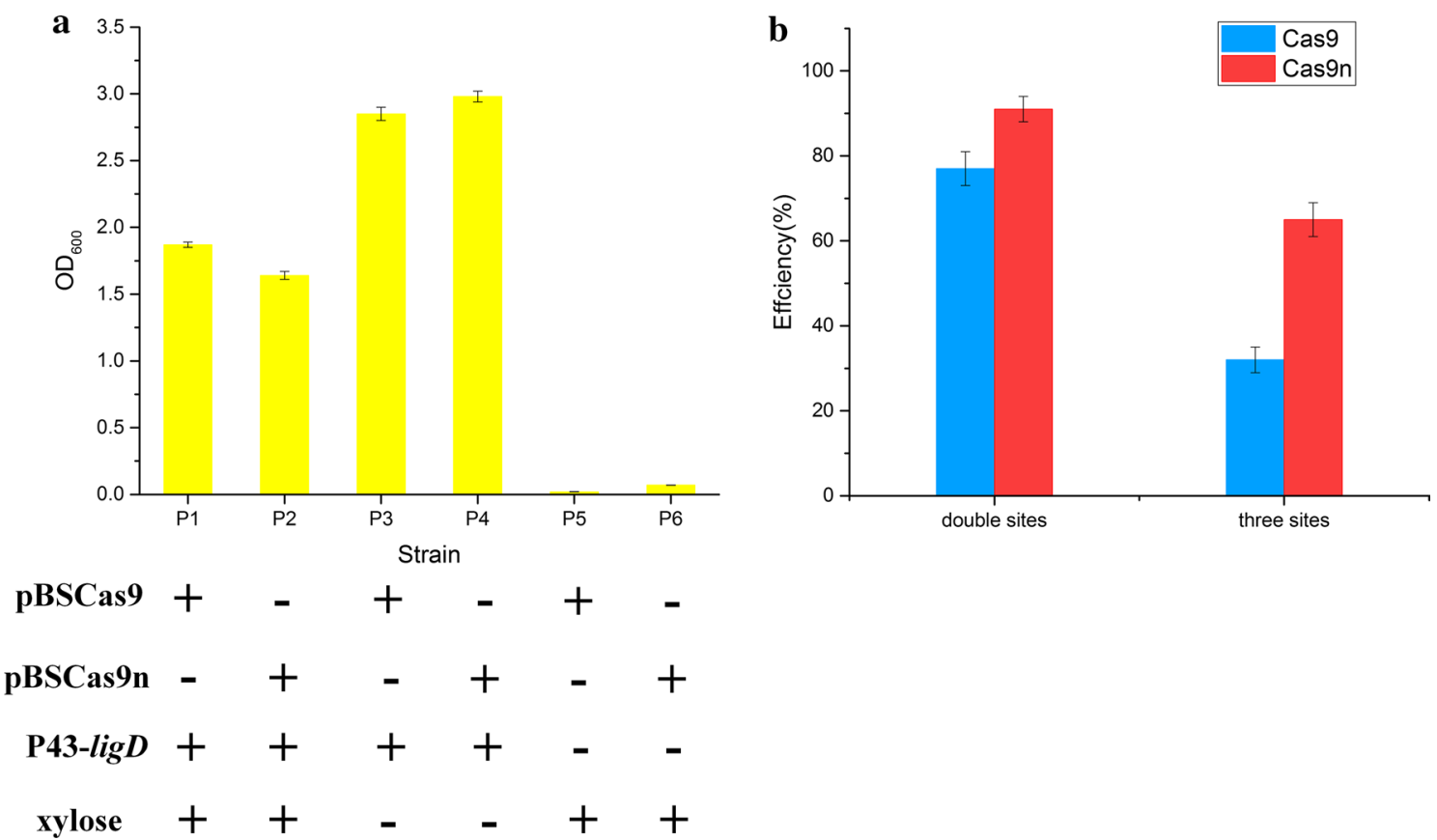

Fig. 6 Strategy for improving CRISPR-Cas9/Cas9n mediated gene editing by regulating ligD. a Cell growths of strains with CRISPR-Cas9/Cas9n mediated gene editing under different induction conditions. Promoter P43 was used to overexpress ligD. The amyE was targeted for Cas9/ Cas9n-induced genome cleavage in this study. $\mathbf{b}$ Editing efficiency for multiplex point mutations by the improved CRISPR-Cas9/Cas9n system. In these genetic modifications, 500 bp homologous-arms were used to achieve recombination. All error bars represent the value of standard deviation which were caculated from three repeated experiments

potential secondary structures. Several secondary structures may reduce the binding capacity (or frequency of binding) of Cas9/Cas9n to the gRNA [53]. In this work, we did not implement optimization in term of PAM sites and gRNAs. The three target genes are arranged sequentially in the operon (SubtiList positions of $r i b G$, ribB ribA: $2430.5 \mathrm{~kb}, 2429.4 \mathrm{~kb}, 2428.8 \mathrm{~kb}$ ) (Fig. 7a) and the efficiency of homologous recombination may have decreased due to their close relative position compared to above-test genes (SubtiList positions of amyE, upp sigE: $327.2 \mathrm{~kb}, 3788.1 \mathrm{~kb}, 1604.1 \mathrm{~kb})$. Nonetheless, the multiplexing efficiency still exceeded $50 \%$ in our sample. This indicated that the CRISPR/Cas9n system we developed and optimized had advantages for multiplex genome editing and made it possible to modulate multiple genes on the chromosome simultaneously in metabolic engineering of $B$. subtilis.

\section{Verification of the optimized strains and analysis of their RBS regions}

Based on the results of productions and yields, a total of 20 strains were chosen for verification in shake-flask fermentations. The 14 strains were chosen from the top 20 highest production strains, including 11 strains with three modulated genes (CY17, CY24, CY33, CY18,
CY16, CY46, CY14, CY44, CY49, CY107 and CY23) and 3 strains with two modulated genes (CY15, CY11 and CY6). Besides the above 14 strains, another 6 high yield strains were chosen from the other 30 high production strains (CY41, CY45, CY43, CY47, CY124 and CY79). Compared to BS89, a total of 18 strains were improved in terms of riboflavin production (Fig. 8), and the best strain CY46 produced $1.39 \mathrm{~g} / \mathrm{L}$ riboflavin with a yield of $17.16 \mathrm{mg} / \mathrm{g}$ glucose, which represents a $59 \%$ increase over the control strain BS89.

The RBS regions of the 20 optimized strains were determined by DNA sequencing (Table 1). In bacteria, RBS are effective control elements for translation initiation and thereby protein expression. Previous studies have generated libraries of RBS with the goal of optimizing the function of a genetic system [54, 55]. In our work, the riboflavin operon was optimized to improve riboflavin production. To investigate the reason for this phenomenon, the secondary structure in the $5^{\prime}$-UTR region of the mRNAs of riboflavin operon genes in the 4 highest production strains (CY124, CY79, CY49 and CY46) were simulated using the RNAfold webserver (http://rna.tbi.univie.ac.at/) with default settings. The RBS structures of the high production strains showed similar changes compared to those of strain BS89. The 

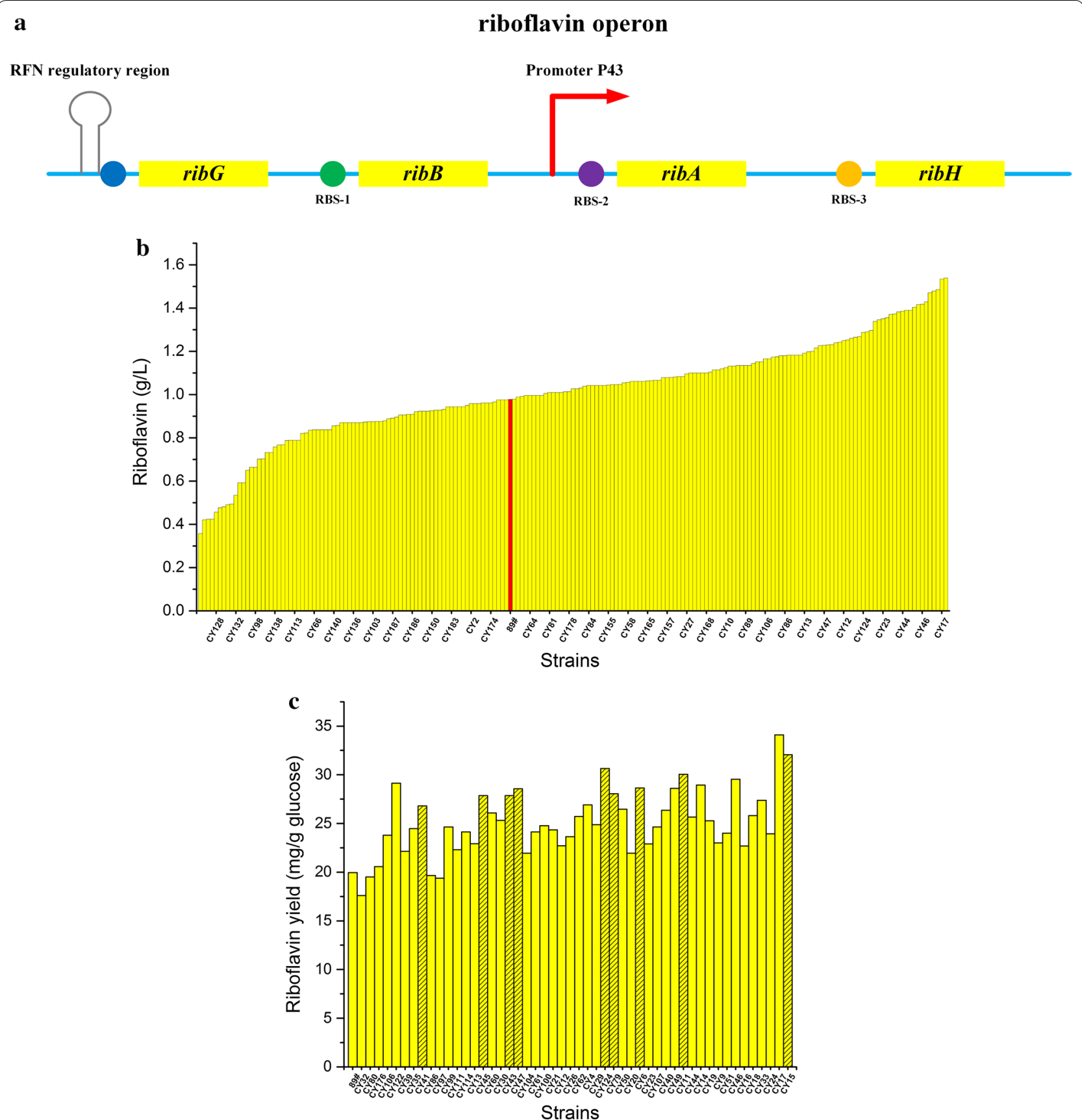

Fig. 7 Optimization of the riboflavin operon by improved CRISPR/Cas9n mediated multiplex gene editing. a Integration of the riboflavin synthesis pathway genes and regulatory elements. b Production of riboflavin in 96-well plates. The red bar shows the control strain. $\mathbf{c}$ Yield of riboflavin in 96-well plates. Striped bar show strains that were selected for secondary screening due to high yields, which were not among the 20 highest-producing strains. The data show the average values and standard deviations of triplicate experiments

results indicated that the RBS of BS89-ribA is hidden in a hairpin structure (Additional file 2: Figure S5), while the RBS of CY46-ribA in the highest production strain CY46 is exposed (Additional file 2: Figure S6). The ribA is a key rate-limiting gene in riboflavin biosynthesis and has a positive direct impact on the production of riboflavin. The hairpin structure covered the RBS of BS89ribA and impeded ribosome binding to the mRNA to start translation, which may explain the low efficiency of this construct. Furthermore, the RBS of BS89-ribH is 


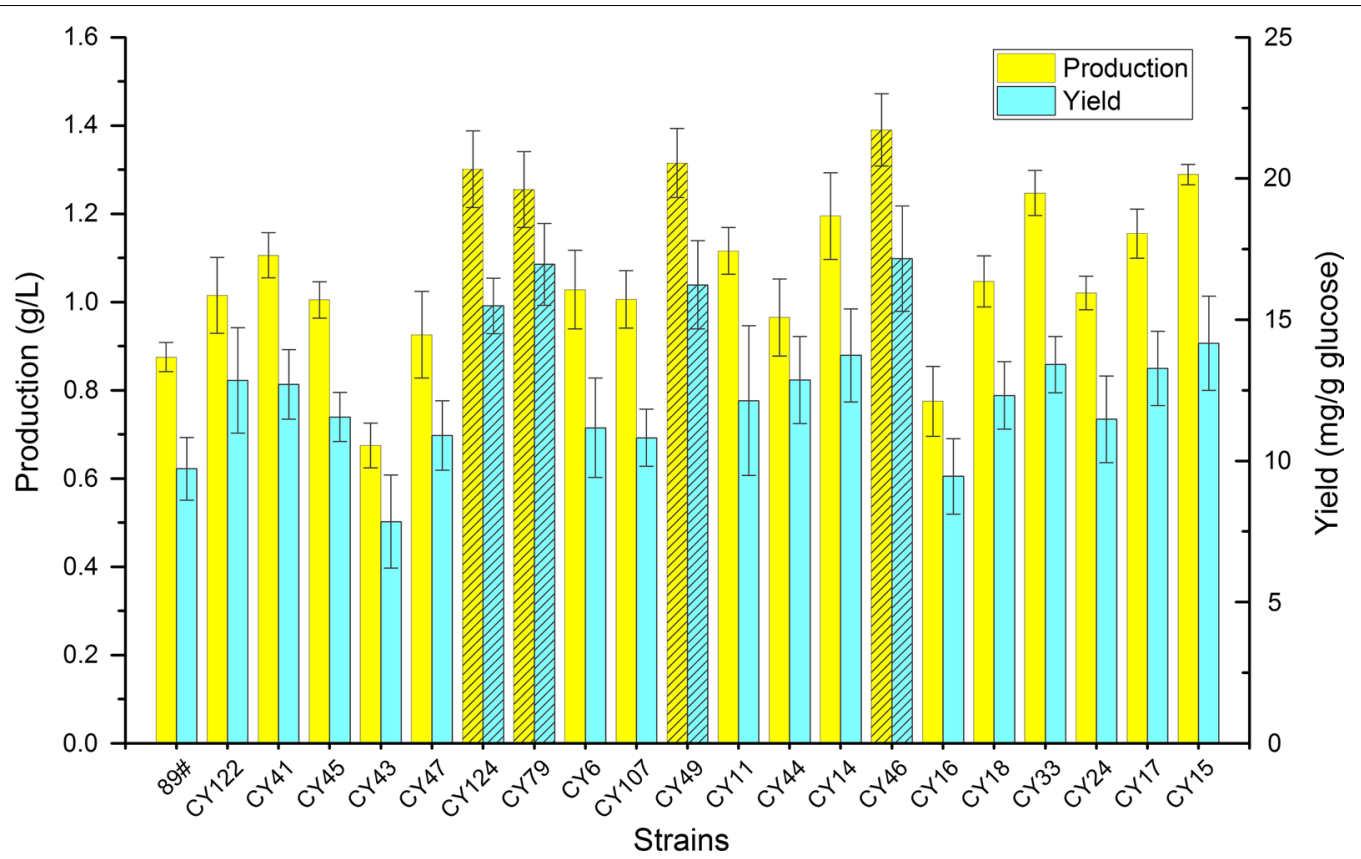

Fig. 8 Productions and yields of riboflavin in shake-flask fermentation. The strains were cultivated aerobically in $50 \mathrm{~mL}$ of YE medium in $500 \mathrm{~mL}$ shake-flask at $240 \mathrm{rpm}$ and $41^{\circ} \mathrm{C}$ with an initial inoculum of $2 \%(\mathrm{v} / \mathrm{v})$. Striped bar indicate strains for which the $5^{\prime}$-UTR regions of the mRNA secondary structure of riboflavin operon genes were simulated. The data are the average values and standard deviations from triplicate experiments

Table 1 Sequences in RBS regions of the riboflavin operon genes of the 20 optimized strains

\begin{tabular}{llll}
\hline Strain & RBS region of $\boldsymbol{r i b \boldsymbol { B }}$ & RBS region of $\boldsymbol{r i b \boldsymbol { A }}$ & RBS region of $\boldsymbol{r i b \boldsymbol { H }}$ \\
\hline CY23 & ATATAAG & GTAAGA & AAGAAGG \\
CY41 & ATTAGAG & AAGGGGA & AGTGAGA \\
CY45 & ATGGTGA & AGGTGGA & ATGGGAA \\
CY43 & AGACAGG & GGCTGGA & AGATGAG \\
CY47 & AACATAG & AAGGAGG & ATAGAGA \\
CY124 & AATGAGA & ACAAGGA & AGGATGG \\
CY79 & AGTAAGA & AAAGGAG & GTAGGAA \\
CY6 & ATGGTGA & ---GAGG & AGTAGAA \\
CY107 & GGAGAGG & ATTGGAG & AAGAGGA \\
CY49 & ATTGAGG & AGTCGAA & GTAAAAG \\
CY11 & AGTCGAA & AAGGAGG & ---GGAG \\
CY44 & GGGAGAA & ATGTGAA & GGCTAGG \\
CY14 & GGAGTAG & GATGGAA & AGTTGAG \\
CY46 & AGTTGAG & AAAGAGA & AAGATAA \\
CY16 & AATGGAG & AATGGAG & AGTTGAG \\
CY18 & GCGAAGG & AGCGAGG & ATGGGAG \\
CY33 & AGGGAGA & AATGGAG & GCGAAGG \\
CY24 & GTTGAAG & AGGAGAA & AAGAAAG \\
CY17 & AAAGAGG & ATTGGAG & AGCAGGA \\
CY15 & AGGAGGA & AAGGAGG & AATGGAG \\
\hline
\end{tabular}

a The Wild type RBSs are shown in bold

b "_" indicates base deletion in this location located in a loop structure (Additional file 2: Figure S5), while the RBS of CY46-ribH is more exposed (Additional file 2: Figure S6). In contrast to the improved expression of $r i b A$ and $r i b H$, the translational levels of $r i b B$ were decreased in the strain CY46. The RBS of BS89-ribB is more exposed than the RBS of CY46-ribB (Additional file 2: Figure S5 and Figure S6). This demonstrated that the optimized expression of the operon depends on the balanced expression of various genes rather than on overexpressing every gene monotonously. The corresponding RBS sequences of strains CY124, CY79 and CY49 mainly obeyed the described structure laws, except for the RBSs of CY79-ribH and CY124-ribH (Additional file 2: Figure S7, Figure S8 and Figure S9).

\section{Conclusions}

In this study, we developed a CRISPR/Cas9n based method for multiplex and iterative genome editing in $B$. subtilis. We performed a detailed characterization of the method and achieved excellent editing efficiency for various types of modifications, especially for large DNA fragment deletions and multiplex point mutations. We also further improved the editing efficiency of CRISPR/Cas9n mediated multiplexing by inhibiting nicks re-ligation. The gene $\operatorname{lig} D$ was targeted to regulate the nick repair mechanism and thus improve multiplex genome editing. The efficiency for three-point mutations by CRISPR/ Cas9n mediated multiplexing system reached $65 \%$ in the 
ligD knockout strain. Finally, we applied CRISPR/Cas9n mediated multiplex gene editing to optimize the riboflavin operon for enhanced production of riboflavin in $B$. subtilis. The use of the improved CRISPR/Cas9n system made it possible to modulate multiple genes for metabolic engineering in $B$. subtilis. To our best knowledge, our work offers not only the iterative CRISPR/Cas9n system for $B$. subtilis but also the highest efficiency for simultaneous modulation of multiple genes on the chromosome in B. subtilis reported to date.

\section{Methods}

\section{Strains and culture conditions}

All bacterial strains and plasmids used in this work are listed in Table 2. E. coli. DH5 $\alpha$ was used as the host strain for cloning and plasmid construction. B. subtilis 168 was used for the characterization of CRISPRCas9/Cas9n mediated genome editing in cells. All other $B$. subtilis strains were derived from the wild-type $B$. subtilis168. Luria-Bertani (LB) medium was used for plasmid construction in E. coli. Riboflavin production was tested in $\mathrm{YE}$ medium $\left(\mathrm{K}_{2} \mathrm{HPO}_{4} 0.5 \mathrm{~g} / \mathrm{L}, \mathrm{KH}_{2} \mathrm{PO}_{4}\right.$ $0.5 \mathrm{~g} / \mathrm{L},\left(\mathrm{NH}_{4}\right)_{4} \mathrm{SO}_{4} 0.5 \mathrm{~g} / \mathrm{L}$, yeast extract $2 \mathrm{~g} / \mathrm{L}$, glucose $100 \mathrm{~g} / \mathrm{L}$ ). The transformation of $B$. subtilis was performed using a standard protocol for natural competence [56]. Transformed cells were cultivated on LB agar plates at $37^{\circ} \mathrm{C}$ for $24 \mathrm{~h}$. During strain construction, the cultures were grown aerobically at $37{ }^{\circ} \mathrm{C}$ in LB medium. When required, antibiotics were added to the media at the following concentrations: $100 \mu \mathrm{g} / \mathrm{mL}$ ampicillin and $10 \mu \mathrm{g} /$ $\mathrm{mL}$ chloramphenicol for $E$. coli selection; $5 \mu \mathrm{g} / \mathrm{mL}$ chloramphenicol and $0.5 \mu \mathrm{g} / \mathrm{mL}$ erythromycin for $B$. subtilis selection. All colonies were collected and stored at $-80{ }^{\circ} \mathrm{C}$ as a $15 \%$ glycerol stock.

\section{Plasmids construction}

To construct the plasmid pBSCas9 (Additional file 1: Figure S1), the promoter $\mathrm{P} 43$ was amplified from the genome of B. subtilis 168 using the primers P43-F and $\mathrm{P} 43-\mathrm{L}$, and the pUC18 origin was amplified from plasmid pUC18 using the primers pUC-F and pUC-L. The fragments encoding $\mathrm{P} 43$ and the pUC18 replicon were fused using the primers P43-F and pUC-L to generate fragment F1. In this process, the sequence for gRNA-Cas9 binding and a terminator derived from Streptococcus pyogenes were synthesized as part of the primers and introduced into the plasmid pBSCas9 backbone. The cat gene was amplified from plasmid pHP13 using the primers $\mathrm{Cm}-\mathrm{F}$ and $\mathrm{Cm}-\mathrm{L}$, the repF replicon was amplified from pEBscop1 using the primers repF-F and repF-L, the PxylA cassette was amplified from pAX01 using the primers $x y l A-\mathrm{F}$ and $x y l A-\mathrm{L}$. The fragments encoding the cat gene, repF replicon and PxylA cassette were fused using the primers pUC-F and xylA-L to generate fragment F2. The Cas9 gene was amplified from pCas9cur using the primers Cas9-F and Cas9-L to generate fragment F3. The fragments F1, F2 and F3 were assembled using the CPEC method [57] to generate pBSCas9. To construct the targeting gRNA, a set of primers was used to PCR amplify the pBSCas9 backbone. The 20 bp spacer sequence specific for each target was synthesized as part of the primers. The PCR product was then self-ligated using Golden Gate Assembly [58] to obtain the corresponding plasmid pBSCas9-gRNA. We employed a previously described method to use a single Golden Gate assembly reaction to construct the gRNA plasmid expressing two or three gRNAs simultaneously [21]. The detailed design and procedure are shown in Additional file 2: Figure S2.

To construct the plasmid pDonor (Additional file 1: Figure S1), the backbone of the plasmid pHP13 was amplified iteratively using the primers pairs $\mathrm{pDon} 1-\mathrm{F} / \mathrm{L}$ and pDon2-F/L. In this process, the promoter Pmanp and the sequence for gRNA targeting rep60 replicon were synthesized as part of the primers and introduced, respectively, into pHP13 backbone. The PCR product from each step of PCR was ligated using Golden Gate Assembly, finally resulting in pDonor. To construct the donor DNA, homologous arms flanking the target loci and sequence to be inserted were separately amplified and were then fused by overlap-extension PCR. The fused fragment was introduced into pDonor using the CPEC method.

To construct the Cas9 nickase, the backbone of the plasmid pBSCas9 was amplified using $5^{\prime}$-phosphorylated primers and was then ligated by T4 DNA ligase. In this process, the mutations D10A and H840A were synthesized as part of the primers and introduced, respectively, into the pBSCas9 backbone. All primers and spacers used in this study are listed in Additional file 2: Table S1.

\section{Iterative genome editing procedure}

The competent cells of B. subtilis 168 were prepared using previously described method. The two plasmids, respectively, expressing gRNA and donor DNA were introduced into B. subtilis successively. The transformants were seeded into LB medium containing chloramphenicol $(5 \mu \mathrm{g} / \mathrm{mL})$, erythromycin $(0.5 \mu \mathrm{g} / \mathrm{mL})$ and $1 \%$ xylose and cultivated for $8-10 \mathrm{~h}$. The cultured cells were serially diluted and spread on LB agar plate containing chloramphenicol $(5 \mu \mathrm{g} / \mathrm{mL})$ and erythromycin $(0.5 \mu \mathrm{g} /$ $\mathrm{mL})$. The mutations were confirmed by phenotypic validation, PCR and DNA sequencing. For curing the plasmid pDonor, the positive colony was inoculated in LB medium containing $1 \%$ xylose and $1 \%$ mannose and cultivated for $12 \mathrm{~h}$ (Additional file 2: Figure S3). Then, the colonies that were sensitive to erythromycin were cultured at $50{ }^{\circ} \mathrm{C}$, overnight for curing plasmid pBSCas 9 or 
Table 2 Strains and plasmids used in this study

\begin{tabular}{|c|c|c|}
\hline Name & Relevant genotype & Source/reference \\
\hline \multicolumn{3}{|l|}{ Strains } \\
\hline B. subtilis 168 & Wild-type strain, $\operatorname{trp} C 2$ & $\mathrm{BGSC}^{\mathrm{a}}$ \\
\hline B. subtilis $168 \triangle$ ligD & B. subtilis 168 $\triangle$ ligD & This study \\
\hline BS89 & $\begin{array}{l}\text { B. subtilis } 168 \Delta u p p \text { ribC*ribO* } \\
\text { yhcF } F^{*} y v r H^{*} y w a A^{*} \text { P43::ribA }\end{array}$ & {$[55]$} \\
\hline BS89 8 ligD & BS89 $\triangle l i g D$ & This study \\
\hline CY series strains & Modulation library strains generated with CRISPR/Cas9n technique & This study \\
\hline E. coli. DH5a & 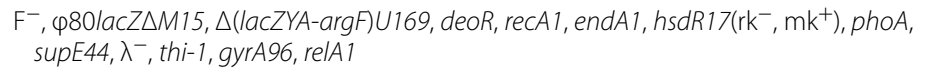 & Lab stock \\
\hline \multicolumn{3}{|l|}{ Plasmids } \\
\hline pCas9cur & E. coli cloning vector for the express Cas9 protein, $\mathrm{Amp}^{R}$ & Lab stock \\
\hline pBSCas9 & $\begin{array}{l}\text { B. subtilis/E. coli shuttle vector, } \mathrm{Cm}^{\mathrm{R}} \\
\mathrm{P}_{x y / A^{-} \text {Cas9, P43-gRNA }}\end{array}$ & This study \\
\hline pBSCas9n & pBSCas9 harboring Cas9 mutation & This study \\
\hline pDonor & $\begin{array}{l}\text { B. subtilis/E. coli shuttle vector, } \mathrm{Cm}^{\mathrm{R}} \\
\text { Amp }\end{array}$ & This study \\
\hline $\mathrm{pHG}$ & Derived from pUC18 for multiple gRNA construction & This study \\
\hline pUC18 & E. coli cloning vector, Amp ${ }^{R}$ & Lab stock \\
\hline pEBs-cop 1 & B. subtilis/E. coli shuttle vector, $\mathrm{Em}^{\mathrm{R}}, \mathrm{Amp}^{\mathrm{R}}$ & Lab stock \\
\hline pAX01 & B. subtilis integration vector, $P_{x y / A} E m^{R}, A m p^{R}$ & Lab stock \\
\hline $\mathrm{pHP13}$ & B. subtilis/E.coli shuttle vector, $\mathrm{Cm}^{\mathrm{R}}, \mathrm{Em}^{\mathrm{R}}$ & Lab stock \\
\hline pBSCas9-amyEgRNA & Derived from pBSCas9 for targeting amyE & This study \\
\hline pBSCas9n-amyEgRNA & Derived from pBSCas9n for targeting amyE & This study \\
\hline pBSCas9-Ins1 kbamyEgRNA & Derived from pBSCas9 for targeting $\triangle 1 \mathrm{kbamy} E$ & This study \\
\hline pBSCas9n-Ins1kbamyEgRNA & Derived from pBSCas9n for targeting $\triangle 1 \mathrm{kbamy} E$ & This study \\
\hline pBSCas9-Ins2kbamyEgRNA & Derived from pBSCas9 for targeting $\triangle 2 \mathrm{kbamy} E$ & This study \\
\hline pBSCas9n-Ins2kbamyEgRNA & Derived from $\mathrm{pBSCas} 9 \mathrm{n}$ for targeting $\triangle 2 \mathrm{~kb} a m y E$ & This study \\
\hline pBSCas9-amyE/uppgRNA & Derived from pBSCas9 for targeting amyE and upp & This study \\
\hline pBSCas9n-amyE/uppgRNA & Derived from pBSCas9n for targeting amyE and upp & This study \\
\hline pBSCas9-amyE/upp/sigEgRNA & Derived from pBSCas9 for targeting amyE,upp and sigE & This study \\
\hline pBSCas9n-amyE/upp/sigEgRNA & Derived from pBSCas9n for targeting amyE,upp and sigE & This study \\
\hline pBSCas9-LGgRNA & Derived from pBSCas9 for targeting large DNA fragment & This study \\
\hline pBSCas9n-LGgRNA & Derived from pBSCas9n for targeting large DNA fragment & This study \\
\hline pDonor-Del1 kb & Derived from pDonor for $1 \mathrm{~kb}$ deletion & This study \\
\hline pDonor-Del2kb & Derived from pDonor for $2 \mathrm{~kb}$ deletion & This study \\
\hline pDonor-Del4kb & Derived from pDonor for $4 \mathrm{~kb}$ deletion & This study \\
\hline pDonor-Del6kb & Derived from pDonor for $6 \mathrm{~kb}$ deletion & This study \\
\hline pDonor-Del8kb & Derived from pDonor for 8 kb deletion & This study \\
\hline pDonor-Ine1kb & Derived from pDonor for $1 \mathrm{~kb}$ insertion & This study \\
\hline pDonor-Ine2kb & Derived from pDonor for $2 \mathrm{~kb}$ insertion & This study \\
\hline pDonor-DelLDNA & Derived from pDonor for large DNA fragment deletion & This study \\
\hline pDonor-MuamyE & Derived from pDonor for codon replacement in amyE & This study \\
\hline pDonor-MuamyE/upp & Derived from pDonor for codon replacement in amyE and upp & This study \\
\hline pDonor-MuamyE/upp/sigE & Derived from pDonor for codon replacement in amyE,upp and sigE & This study \\
\hline pBSCas9n-gRNArib & Derived from pBSCas9n for targeting ribB, ribA and ribH & This study \\
\hline pDonor-ribRBSLib & Derived from pDonor for generating a combinatorial library & This study \\
\hline
\end{tabular}

Cm chloramphenicol; $A m p$ ampicillin; Em erythromycin; $R$ resistance

a Bacillus Genetic Stock Center 
pBSCas9n [16]. After plasmid curing, the cultures were streaked and colonies were tested for chloramphenicol sensitivity. To save time, we usually inoculated colonies for the next round of editing before testing for chloramphenicol sensitivity because of the high curing efficiency in this step.

\section{Determining the editing efficiency and number of colony forming units}

The $a m y E$ gene locus was used as the target for gene knockout, gene insertion and single-point mutation. The $a m y E$ and $u p p$ genes were targeted for two-point mutations, amyE, upp and sigE were targeted for three simultaneous point mutations. The prophage (-like) region (SubtiList coordinates: 528148-548697) was targeted for large DNA fragment deletion. The editing efficiency was determined by calculating the PCR analytical number of positive colonies. To save time, when two or three-point mutations were implemented, phenotypic analysis ( $a m y E$ and $u p p$ ) was employed to exclude false-positive colonies and then DNA sequencing was performed to confirm that the target genes were modified.

The total number of cells were counted after the xyloseinduced cells harboring two plasmids were diluted and spread onto LB agar plates. The CFU were determined by calculating counts per $1 \mathrm{~mL}$ based on total liquid volume spread on plates.

\section{Generation of an RBS-modulation library using CRISPR/ Cas9n}

The RBS of ribA was designed as a typical sequence AAGGAGG in B. subtilis, and it was introduced into the genome during the construction of strain BS89. The RBS sequences of $r i b B$ and $r i b H$ were determined by sequence analysis of non-coding region upstream of their start codons. They were identified with high homology to GGAGG at about 4 bp upstream of the start codon [59]. To generate a combinatorial library of variably improved riboflavin operon pathway genes, the plasmids pBSCas9n-gRNArib and pDonor-ribRBSLib were constructed. To preserve RBS sequence specificity and basal translation strength in $B$. subtilis, the first one and last two bases were designed as A or G [59], and the others were degenerate. The RBS library was correspondingly designed as semi-degenerate sequences RNNNNRR (N: $25 \%$ possibility of each of the A, G, C, and T bases; R: $50 \%$ possibility of each of the A and G bases). The RBS library was designed based on donor DNA with random nucleotides (RNNNNRR) in the RBS region. The random nucleotides were provided on primers used to construct the donor DNA plasmid. The upstream and downstream fragments of corresponding genes were obtained by PCR amplification with the genome as template. DNA fragments containing the regulator library were obtained by overlap-extension PCR with upstream and downstream fragments and inserted into $\mathrm{pDonor}$ to form $\mathrm{pDo}$ nor-ribRBSLib using the CPEC method.

\section{Fermentation conditions}

Riboflavin production was carried out in shake-flask cultivation medium. To test the riboflavin biosynthesis activity of the engineered strains, a single colony of each strain was transferred into $5 \mathrm{~mL}$ of LB medium and incubated at $41^{\circ} \mathrm{C}$ in a rotatory shaker at $240 \mathrm{rpm}$ for $14 \mathrm{~h}$ to prepare the inocula. The inocula were added aseptically at $2 \%(\mathrm{v} / \mathrm{v})$ to a $500 \mathrm{~mL}$ shake flask containing $50 \mathrm{~mL}$ of shake-flask YE medium. The fermentation was carried out at $41{ }^{\circ} \mathrm{C}$ in shake flasks at $240 \mathrm{rpm}$ for $48 \mathrm{~h}$.

\section{Analytical methods}

The growth of $B$. subtilis was monitored by measuring the optical density at $600 \mathrm{~nm}\left(\mathrm{OD}_{600}\right)$ using a conventional UV-Vis spectrophotometer. Glucose consumption was quantified using an SBA-40E biosensor (Shandong Province Academy of Sciences, China). For riboflavin measurement, samples were first diluted with $0.05 \mathrm{M}$ $\mathrm{NaOH}$ and centrifuged at $1000 \mathrm{~g}$ for $2 \mathrm{~min}$ to remove the cells, after which the supernatant was diluted with acetic acid sodium-acetate buffer ( $\mathrm{pH}$ 5.0) to the linear range of the spectrophotometer and the absorbance at $444 \mathrm{~nm}$ was recorded [60]. The riboflavin concentration was calculated using the standard equation which had been validated, $Y=\left(\mathrm{A}_{444}-0.0057\right) \times \mathrm{DF} / 0.0321\left[R^{2}=0.9968 ; Y\right.$, the riboflavin concentration of sample $(\mathrm{mg} / \mathrm{L}) ; \mathrm{A}_{444}$, the value of absorbance at $444 \mathrm{~nm}$; $\mathrm{DF}$, dilution fold; $\mathrm{A}_{444}$ was controlled within the range of $0.3-0.8$ by dilution].

\section{Additional files}

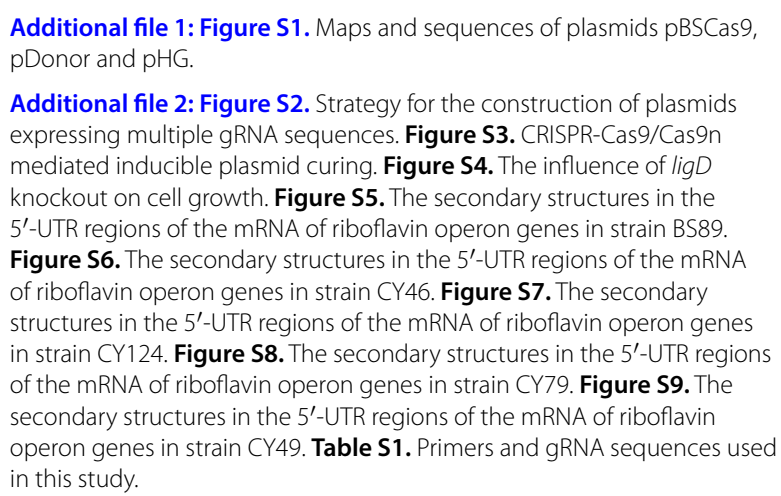

Additional file 1: Figure S1. Maps and sequences of plasmids pBSCas9, pDonor and $\mathrm{pHG}$.

Additional file 2: Figure S2. Strategy for the construction of plasmids expressing multiple gRNA sequences. Figure S3. CRISPR-Cas9/Cas9n mediated inducible plasmid curing. Figure S4. The influence of ligD knockout on cell growth. Figure S5. The secondary structures in the 5'-UTR regions of the mRNA of riboflavin operon genes in strain BS89. Figure S6. The secondary structures in the 5'-UTR regions of the mRNA of riboflavin operon genes in strain CY46. Figure S7. The secondary structures in the $5^{\prime}$-UTR regions of the mRNA of riboflavin operon genes in strain CY124. Figure S8. The secondary structures in the 5'-UTR regions of the mRNA of riboflavin operon genes in strain CY79. Figure S9. The secondary structures in the 5'-UTR regions of the mRNA of riboflavin operon genes in strain CY49. Table S1. Primers and gRNA sequences used in this study.

Abbreviations

DSB: double-strand break; SSB: single-strand break; HDR: homology directed repair; CFU: colony forming units; NHEJ: non-homologous end joining; CRISPR: 
clustered regularly interspaced short palindromic repeats; GRAS: generally regarded as safe.

\section{Authors' contributions}

$\mathrm{DL}, \mathrm{CH}$ and $\mathrm{ZW}$ designed the experiments; $\mathrm{DL}, \mathrm{CH}$, JG and $\mathrm{PZ}$ performed the experiments and analyzed the results; $D L, T C$ and $Z W$ wrote the manuscript; $Z W$, TC and XZ supervised the work. All authors read and approved the final manuscript.

\section{Funding}

This work was supported by the National Key Research and Development Program of China (2018YFA0901300) and National Natural Science Foundation of China (NSFC-21576200, NSFC-21576191 and NSFC-21621004).

\section{Availability of data and materials}

The materials and data used and/or analyzed during the current study are available from the corresponding author on reasonable request.

\section{Ethics approval and consent to participate}

Not applicable.

\section{Consent for publication}

Not applicable.

\section{Competing interests}

The authors declare that they have no competing interests.

Received: 19 April 2019 Accepted: 4 August 2019

Published online: 27 September 2019

\section{References}

1. Choi KR, Lee SY. CRISPR technologies for bacterial systems: Current achievements and future directions. Biotechnol Adv. 2016;34(7):1180-209.

2. Jakociunas T, Jensen MK, Keasling JD. CRISPR/Cas9 advances engineering of microbial cell factories. Metab Eng. 2016;34:44-59.

3. Yadav VG, De Mey M, Giaw Lim C, Kumaran Ajikumar P, Stephanopoulos $G$. The future of metabolic engineering and synthetic biology: towards a systematic practice. Metab Eng. 2012;14(3):233-41.

4. Judith B, Christoph W. Advanced biotechnology: metabolically engineered cells for the bio-based production of chemicals and fuels, materials, and health-care products. Angew Chem Int Ed Engl. 2015;46(18):3328-50.

5. Paddon CJ, Keasling JD. Semi-synthetic artemisinin: a model for the use of synthetic biology in pharmaceutical development. Nat Rev Microbiol. 2014;12(5):355.

6. Lee ME, Anil A, Han AS, Tomlin CJ, Dueber JE. Expression-level optimization of a multi-enzyme pathway in the absence of a high-throughput assay. Nucleic Acids Res. 2013;41(22):10668-78.

7. Lanza AM, Curran KA, Rey LG, Alper HS. A condition-specific codon optimization approach for improved heterologous gene expression in Saccharomyces cerevisiae. BMC Syst Biol. 2014;8(1):33.

8. Liu DY, Mao ZT, Guo JX, Wei LY, Ma HW, Tang YJ, Chen T, Wang ZW, Zhao XM. Construction, Model-Based Analysis, and Characterization of a Promoter Library for Fine-Tuned Gene Expression in Bacillus subtilis. ACS Synth Biol. 2018;7(7):1785-97.

9. Liu YF, Li JH, Du GC, Chen J, Liu L. Metabolic engineering of Bacillus subtilis fueled by systems biology: recent advances and future directions. Biotechnol Adv. 2017;35(1):20-30.

10. Wang HH, Isaacs FJ, Carr PA, Sun ZZ, Xu G, Forest CR, Church GM. Programming cells by multiplex genome engineering and accelerated evolution. Nature. 2009:460(7257):894-8

11. Dicarlo JE, Conley AJ, Penttilä M, Jäntti J, Wang HH, Church GM. Yeast Oligo-mediated Genome Engineering (YOGE). Acs Synthetic Biology. 2013;2(12):741-9.

12. Warner JR, Reeder PJ, Anis KF, Woodruff LBA, Gill RT. Rapid profiling of a microbial genome using mixtures of barcoded oligonucleotides. Nat Biotechnol. 2010;28(8):856-62.
13. Santos CNS, Regitsky DD, Yoshikuni Y. Implementation of stable and complex biological systems through recombinase-assisted genome engineering. Nature Communications. 2013;4(9):2503.

14. Garst AD, Bassalo MC, Pines G, Lynch SA, Halwegedwards AL, Liu R, Liang L, Wang Z, Zeitoun R, Alexander WG. Genome-wide mapping of mutations at single-nucleotide resolution for protein, metabolic and genome engineering. Nat Biotechnol. 2017:35(1):48.

15. Bao Z, Hamedirad M, Xue P, Xiao H, Tasan I, Chao R, Liang J, Zhao H. Genome-scale engineering of Saccharomyces cerevisiae with singlenucleotide precision. Nat Biotechnol. 2018;36(6):505-8.

16. Shi T, Wang G, Wang Z, Fu J, Chen T, Zhao X. Establishment of a markerless mutation delivery system in Bacillus subtilis stimulated by a double-strand break in the chromosome. PLoS ONE. 2013:8(11):e81370.

17. Alain B, Patrice F, Andy C, Aurore C, Bernard J. New integrative method to generate Bacillus subtilis recombinant strains free of selection markers. Appl Environ Microbiol. 2004;70(12):7241.

18. Xiao-Zhou Z, Xin Y, Zhong-Li C, Qing H, Shun-Peng L. mazF, a novel counter-selectable marker for unmarked chromosomal manipulation in Bacillus subtilis. Nucleic Acids Res. 2006;34(9):e71.

19. Wang Y, Weng J, Waseem $R$, Yin X, Zhang R, Shen Q. Bacillus subtilis genome editing using SsDNA with short homology regions. Nucleic Acids Res. 2012:40(12):e91.

20. Peters JM, Silvis MR, Zhao D, Hawkins JS, Gross CA, Qi LS. Bacterial CRISPR: accomplishments and prospects. Curr Opin Microbiol. 2015:27:121-6

21. Li Y, Lin Z, Huang C, Zhang Y, Wang Z, Tang YJ, Chen T, Zhao X. Metabolic engineering of Escherichia coli using CRISPR-Cas9 meditated genome editing. Metab Eng. 2015;31:13-21.

22. Jiang Y, Chen B, Duan C, Sun B, Yang J, Yang S. Multigene editing in the Escherichia coli genome via the CRISPR-Cas9 system. Appl Environ Microbiol. 2015:81(7):2506-14.

23. Wang H, La Russa M, Qi LS. CRISPR/Cas9 in genome editing and beyond. Annu Rev Biochem. 2016;85:227-64.

24. Lu Z, Yang S, Yuan X, Shi Y, Ouyang L, Jiang S, Yi L, Zhang G. CRISPRassisted multi-dimensional regulation for fine-tuning gene expression in Bacillus subtilis. Nucleic Acids Res. 2019. https://doi.org/10.1093/nar/ gkz072.

25. Gaj T, Gersbach CA, Barbas CF 3rd. ZFN, TALEN, and CRISPR/ Cas-based methods for genome engineering. Trends Biotechnol. 2013;31(7):397-405.

26. Cong L, Ran FA, Cox D, Lin S, Barretto R, Habib N, Hsu PD, Wu X, Jiang W, Marraffini L. Multiplex genome engineering using CRISPR/Cas systems. Science. 2013;339:1231143.

27. Luther D, Nancy M. DNA nicks promote efficient and safe targeted gene correction. Plos ONE. 2011;6(9):e23981.

28. Michael JM, Audrey M-S, Barry LS, Dusty AM. Single-strand nicks induce homologous recombination with less toxicity than doublestrand breaks using an AAV vector template. Nucleic Acids Res. 2011:39(3):926-35.

29. Zerbini F, Zanella I, Fraccascia D, Konig E, Irene C, Frattini LF, Tomasi M, Fantappie L, Ganfini L, Caproni E, et al. Large scale validation of an efficient CRISPR/Cas-based multi gene editing protocol in Escherichia coli. Microb Cell Fact. 2017;16(1):68.

30. Song X, Huang H, Xiong Z, Ai L, Yang S. CRISPR-Cas 9(D10A) nickaseassisted genome editing in Lactobacillus casei. Appl Environ Microbiol. 2017;83(22):e01259-317.

31. Li Q, Chen J, Minton NP, Zhang Y, Wen Z, Liu J, Yang H, Zeng Z, Ren X, Yang $J$, et al. CRISPR-based genome editing and expression control systems in Clostridium acetobutylicum and Clostridium beijerinckii. Biotechnol J. 2016;11(7):961-72.

32. Li K, Cai D, Wang Z, He Z, Chen S. Development of an efficient genome editing tool in Bacillus licheniformis using CRISPR-Cas9 nickase. Appl Environ Microbiol. 2018;84(6):AEM.02608-17.

33. Altenbuchner J. Editing of the Bacillus subtilis Genome by the CRISPRCas9 System. Appl Environ Microbiol. 2016;82(17):5421-7.

34. Westbrook AW, Moo-Young M, Chou CP. Development of a CRISPR-Cas9 Tool Kit for Comprehensive Engineering of Bacillus subtilis. Appl Environ Microbiol. 2016:82(16):4876-95.

35. So Y, Park SY, Park EH, Park SH, Kim EJ, Pan JG, Choi SK. A highly efficient CRISPR-Cas9-mediated large genomic deletion in Bacillus subtilis. Front Microbiol. 2017:8:1167. 
36. Xu T, Li Y, Shi Z, Hemme CL, LiY ZhuY Van Nostrand JD, He Z, Zhou J. Efficient genome editing in Clostridium cellulolyticum via CRISPR-Cas9 nickase. Appl Environ Microbiol. 2015;81(13):4423-31.

37. Toymentseva AA, Altenbuchner J. New CRISPR-Cas9 vectors for genetic modifications of Bacillus species. FEMS Microbiol Lett. 2019;366(1):fyn284.

38. Zhang K, Duan X, Wu J. Multigene disruption in undomesticated Bacillus subtilis ATCC 6051a using the CRISPR/Cas9 system. Sci Rep. 2016;6:27943.

39. Hong KQ, Liu DY, Chen T, Wang ZW. Recent advances in CRISPR/Cas9 mediated genome editing in Bacillus subtilis. World J Microbiol Biotechnol. 2018;34(10):153

40. Da-Eun J, Seung-Hwan P, Jae-Gu P, Eui-Joong K, Soo-Keun C. Genome engineering using a synthetic gene circuit in Bacillus subtilis. Nucleic Acids Res. 2015;43(6):e42.

41. Morimoto T, Ara K, Ozaki K, Ogasawara N. A simple method for introducing marker-free deletions in the Bacillus subtilis genome. Genes Genet Syst. 2009;84(4):315-8.

42. Satomura A, Nishioka R, Mori H, Sato K, Kuroda K, Ueda M. Precise genome-wide base editing by the CRISPR Nickase system in yeast. Sci Rep. 2017;7(1):2095.

43. Metzger MJ, Mcconnellsmith A, Stoddard BL, Miller AD. Single-strand nicks induce homologous recombination with less toxicity than double-strand breaks using an AAV vector template. Nucleic Acids Res. 2011;39(3):926-35.

44. Zhu X, Zhao D, Qiu H, Fan F, Man S, Bi C, Zhang X. The CRISPR/Cas9-facilitated multiplex pathway optimization (CFPO) technique and its application to improve the Escherichia coli xylose utilization pathway. Metab Eng 2017:43(Pt A):37-45.

45. Doench JG, Fusi N, Sullender M, Hegde M, Vaimberg EW, Donovan KF, Smith I, Tothova Z, Wilen C, Orchard R, et al. Optimized sgRNA design to maximize activity and minimize off-target effects of CRISPR-Cas9. Nat Biotechnol. 2016:34(2):184-91.

46. Cho JS, Choi KR, Prabowo CPS, Shin JH, Yang D, Jang J, Lee SY. CRISPR/ Cas9-coupled recombineering for metabolic engineering of Corynebacterium glutamicum. Metab Eng. 2017;42:157-67.

47. Moeller R, Stackebrandt E, Reitz G, Berger T, Rettberg P, Doherty AJ, Horneck G, Nicholson WL. Role of DNA repair by nonhomologousend joining in Bacillus subtilis spore resistance to extreme dryness, mono- and polychromatic UV, and ionizing radiation. J Bacteriol. 2007;189(8):3306-11.

48. Helleday T, Lo J, Gent DCV, Engelward BP. DNA double-strand break repair: From mechanistic understanding to cancer treatment. DNA Repair. 2007;6(7):923-35
49. Weller GR, Boris K, Rajat R, Tonkin LM, Elizabeth S, Marina D, Susanne Krogh D, Day JP, Adam W, Fabrizio DADF. Identification of a DNA nonhomologous end-joining complex in bacteria. Science. 2002;297(5587):1686-9.

50. Moeller R, Schuerger AC, Reitz G, Nicholson WL. Impact of two DNA repair pathways, homologous recombination and non-homologous end joining, on bacterial spore inactivation under simulated martian environmental conditions. Icarus. 2011;215(1):204-10.

51. Shi T, Wang Y, Wang Z, Wang G, Liu D, Fu J, Chen T, Zhao X. Deregulation of purine pathway in Bacillus subtilis and its use in riboflavin biosynthesis. Microb Cell Fact. 2014;13(1):101.

52. Bao Z, Xiao H, Liang J, Zhang L, Xiong X, Sun N, Si T, Zhao H. Homologyintegrated CRISPR-Cas (HI-CRISPR) system for one-step multigene disruption in Saccharomyces cerevisiae. Acs Synth Biol. 2015;4(5):585-94.

53. Tim W, Wei JJ, Sabatini DM, Lander ES. Genetic screens in human cells using the CRISPR-Cas9 system. Science. 2013;343(6166):80-4.

54. Pfleger B, Pitera D, Smolke DC, Keasling J. Combinatorial engineering of intergenic regions in operons tunes expression of multiple genes. Nat Biotechnol. 2006;24(8):1027.

55. Salis HM, Mirsky EA, Voigt CA. Automated design of synthetic ribosome binding sites to control protein expression. Nat Biotechnol. 2009;27(10):946-50.

56. Anagnostopoulos C, Spizizen J. Requirements for transformation in Bacillus subtilis. J Bacteriol. 1961;81(5):741.

57. Quan JY, Tian JD. Circular polymerase extension cloning for highthroughput cloning of complex and combinatorial DNA libraries. Nature Protocols. 2011;6(2):242.

58. Liang J, Chao R, Abil Z, Bao ZH, Zhao HM. FairyTALE: A High-Throughput TAL Effector SynthesisPlatform. ACS Synthetic Biology. 2013;3(2):67-73.

59. Vellanoweth $\mathrm{RL}$, Rabinowitz JC. The influence of ribosome-binding-site elements on translational efficiency in Bacillus subtilis and Escherichia coli in vivo. Molecular Microbiology. 1992;6(9):1105-14.

60. Wang GL, Shi T, Chen T, Wang XY, Wang YC, Liu DY, Guo JX, Fu J, Feng $\mathrm{LL}$, Wang ZW. Integrated whole-genome and transcriptome sequence analysis reveals the genetic characteristics of a riboflavin-overproducing Bacillus subtilis. Metab Eng. 2018;48:138.

\section{Publisher's Note}

Springer Nature remains neutral with regard to jurisdictional claims in published maps and institutional affiliations.
Ready to submit your research? Choose BMC and benefit from:

- fast, convenient online submission

- thorough peer review by experienced researchers in your field

- rapid publication on acceptance

- support for research data, including large and complex data types

- gold Open Access which fosters wider collaboration and increased citations

- maximum visibility for your research: over 100M website views per year

At BMC, research is always in progress.

Learn more biomedcentral.com/submissions 